\title{
Pricing Formula for Exotic Options with Assets Exposed to Counterparty Risk
}

\author{
Li Yan \\ School of Economic Mathematics, Southwestern University of Finance and Economics, Chengdu 611130, China \\ Correspondence should be addressed to Li Yan; yl@2014.swufe.edu.cn
}

Received 2 November 2016; Revised 24 December 2016; Accepted 13 March 2017; Published 27 March 2017

Academic Editor: Leonid Shaikhet

Copyright (c) 2017 Li Yan. This is an open access article distributed under the Creative Commons Attribution License, which permits unrestricted use, distribution, and reproduction in any medium, provided the original work is properly cited.

\begin{abstract}
This paper gives analytical formulas for lookback and barrier options on underlying assets that are exposed to a counterparty risk. The counterparty risk induces a drop in the asset price, but the asset can still be traded after this default time. A novel technique is developed to valuate the lookback and barrier options by first conditioning on the predefault and the postdefault time and then obtain the unconditional analytic formulas for their prices.
\end{abstract}

\section{Introduction}

Lookback and barrier options are among the most popular path-dependent options traded in exchanges and over-thecounter markets. A standard floating lookback call (put) option gives the holder the right to buy (sell) an asset (e.g., stock, index, exchange rate, and interest rate) at its lowest (highest) price during the life of the contract. In other words, the payoffs of a floating lookback call/put are, respectively, $S_{T}-\min _{0 \leq t \leq T} S_{t}$ and $\max _{0 \leq t \leq T} S_{t}-S_{T}$. A barrier option is a financial derivatives contract that is activated ("in") or deactivated ("out") when the price of the underlying asset crosses a certain level (i.e., a barrier) from above ("down") or below ("up"). Lookback and barrier options have been useful in the "real options" literature. For example, Longstaff [1] approximates the marketability values of a security as a continuoustime lookback option. Barrier options are inherent in the reduced-form approach to credit risk, where the default time of a company is modeled as the first hitting time, or barrier breaching time, of the asset process of the company; see Merton [2], Black and Cox [3], Leland and Toft [4], and Chen and Kou [5]. For a review of the literature of both continuous and discrete lookback options and barrier options, please refer to Kou [6] and the references therein.

In the financial market, a counterparty default usually has important influences in various contexts. In terms of credit spreads, one observes in general a positive jump of the default intensity which is called the contagious jump (see, e.g., Jarrow and $\mathrm{Yu}$ [7]). In terms of asset (or stock) values for a firm, the default of a counterparty will in general induce a drop of its value process (see, e.g., El Karoui et al. [8]). Jiao and Pham [9] analyzed the impact of this counterparty risk on the optimal investment problem. In this paper, we study the impact of the counterparty risk on option pricing problems. In particular, we focus on the pricing of lookback and barrier options when the underlying asset is subject to the default of a counterparty and the instantaneous loss of the asset at the default time.

The explicit valuation of vanilla European options with this counterparty default risk was partly given by Ma et al. [10]. However, the derivation of the analytic formula for pricing lookback and barrier options with this default risk model has not been done in the previous literature. The main difficulty lies in that the distribution of the first passage time is difficult to derive due to the default and the continuous trading of the underlying asset after the default time. We consider the conditional density approach of default, which is particularly suitable to study what goes on after the default and was adopted by Jiao and Pham [9] for the optimal investment problem. We derive the explicit distribution of the first passage time and then obtain the analytic formulas for valuation of the lookback and barrier options. 
The outline of the paper is organized as follows. In Section 2, we introduce the financial models. In Section 3, we derive the distribution of the first passage time and then the formula for pricing lookback options. In Section 4, we derive the formula for pricing barrier options. Conclusions are given in the final section.

\section{Financial Models}

In this section, we consider a financial market model with a risky asset subject to counterparty risk: the dynamics of the risky asset is affected by the possibility of the counterparty defaulting. However, this stock still exists and can be traded after the default.

Let $W=\left(W_{t}\right)_{t \in[0, T]}$ be a Brownian motion with horizon $T<\infty$ on the probability space $(\Omega, \mathscr{G}, P)$ and denote by $\mathbb{F}=$ $\left(\mathscr{F}_{t}\right)_{t \in[0, T]}$ the natural filtration of $W$. Let $\tau$, an almost surely finite nonnegative random variable on $(\Omega, \mathscr{G}, P)$, represent the default time. Before $\tau$, the filtration $\mathbb{F}$ represents the information accessible to the investors. After $\tau$, the investors add this new information $\tau$ to the reference filtration $\mathbb{F}$. Introduce $D_{t}=\mathbf{1}_{\{\tau \leq t\}}, 0 \leq t \leq T$, and let $\mathbb{D}=\left(\mathscr{D}_{t}\right)_{t \in[0, T]}$ be the filtration generated by this jump process, and $\mathbb{G}=$ $\left(\mathscr{G}_{t}\right)_{t \in[0, T]}$ the progressively enlarged filtration $\mathbb{F} \vee \mathbb{D}$, representing the structure of information available for the investors over $[0, T]$.

The stock price process is governed by the following dynamic:

$$
d S_{t}=S_{t-}\left(\mu_{t} d t+\sigma_{t} d W_{t}-\gamma_{t} d D_{t}\right), \quad 0 \leq t \leq T
$$

where $\mu, \sigma$, and $\gamma$ are $\mathbb{G}$-predictable processes. From [11], any $\mathbb{G}$-predictable process $\varphi$ can be written in the form:

$$
\varphi_{t}=\varphi_{t}^{F} \mathbf{1}_{\{t<\tau\}}+\varphi_{t}^{d}(\tau) \mathbf{1}_{\{t \geq \tau\}}, \quad 0 \leq t \leq T,
$$

with $\varphi_{t}^{F} \mathbb{F}$-adapted, and $\varphi_{t}^{d}(\theta)$ is measurable with respect to $\mathscr{F}_{t} \otimes \mathscr{B}\left(R_{+}\right)$, for all $t \in[0, T]$. Therefore, the dynamic (1) can be rewritten as

$$
\begin{aligned}
d S_{t}^{F} & =S_{t}^{F}\left(\mu_{t}^{F} d t+\sigma_{t}^{F} d W_{t}\right), \quad 0 \leq t<\tau, \\
d S_{t}^{d}(\tau) & =S_{t}^{d}(\tau)\left(\mu_{t}^{d}(\tau) d t+\sigma_{t}^{d}(\tau) d W_{t}\right), \quad \tau<t \leq T, \\
S_{\tau}^{d}(\tau) & =S_{\tau-}^{F}\left(1-\gamma_{\tau}^{F}\right),
\end{aligned}
$$

where $\mu_{t}^{F}, \sigma_{t}^{F}, \gamma^{F}, S_{t}^{F}$ are $\mathbb{F}$-adapted processes, and $\mu_{t}^{d}(\tau)$, $\sigma_{t}^{d}(\tau), S_{t}^{d}(\tau)$ are $\mathscr{F}_{t} \otimes \mathscr{B}([0, t))$-measurable functions for all $t \in[0, T]$. The process $\gamma$ represents the (proportional) loss on the stock price induced by the default of the counterparty; by misuse of notation, we shall thus identify $\gamma$ in (1) with the $\mathbb{F}$-adapted process $\gamma^{F}$ in (5). When the counterparty defaults, the drift, and diffusion coefficients $(\mu, \sigma)$ of the stock price switch from $\left(\mu^{F}, \sigma^{F}\right)$ to $\left(\mu^{d}(\tau), \sigma^{d}(\tau)\right)$, the postdefault coefficients may depend on the default time $\tau$. Here for simplicity we assume that

$$
\begin{aligned}
\mu_{t}^{F} & =\mu_{1}, \\
\sigma_{t}^{F} & =\sigma_{1}, \\
\mu_{t}^{d}(\tau) & =\mu_{2}, \\
\sigma_{t}^{d}(\tau) & =\sigma_{2}, \\
\gamma_{\tau}^{F} & =\gamma,
\end{aligned}
$$

where $\mu_{1}, \sigma_{1}$ are nonnegative constants, $\mu_{t}^{d}(\theta), \sigma_{t}^{d}(\theta)$ are only deterministic functions of $\theta \in \mathbb{R}_{+}$, in [9], for example $\mu_{t}^{d}(\theta)=$ $\mu^{F}(\theta / T), \sigma_{t}^{d}(\theta)=\sigma^{F}(2-\theta / T), \theta \in[0, T]$, which have the following economic interpretation. The ratio between the pre- and postdefault rate of return is smaller than one and increases linearly with the default time: the postdefault rate of return drops to zero, when the default time occurs near the initial date and converges to the predefault rate of return, when the default time occurs near the finite investment horizon. We have a similar interpretation for the volatility whose ratio is larger than one, which decreases linearly with the default time, converging to the double (resp., initial) value of the predefault volatility, when the default time goes to the initial (resp., terminal horizon) time. The distribution of $\gamma(\gamma \leq 1)$ is fixed. Moreover $\gamma, \tau$, and $W_{t}$ are independent and $\tau$ is an exponential variable with parameter $\lambda$. For more general set-ups, see Jiao and Pham [9].

Assume that $r$ is a risk-free interest rate. Let us define the $\mathbb{G}$-adapted process

$$
\beta_{t}=\frac{\mu_{t}-r-n \lambda I_{t \leq \tau}}{\sigma_{t}}, \quad 0 \leq t \leq T .
$$

By assuming $\mathbb{E}\left[\exp \left\{\int_{0}^{T}(1 / 2)\left|\beta_{t}\right|^{2} d t<\infty\right\}\right]$, we define a probability measure $\widetilde{P}$ which is equivalent to $P$ on $(\Omega, \mathscr{G})$ with Radon-Nikodym density:

$$
\frac{d \widetilde{P}}{d P}=Z_{T}^{\mathbb{G}}=\exp \left\{-\int_{0}^{T} \beta_{t} d W_{t}-\frac{1}{2} \int_{0}^{T} \beta_{t}^{2} d t\right\},
$$

under which, by Girsanov's theorem, $\widetilde{W}_{t}=W_{t}+\int_{0}^{t} \beta_{u} d u$ is a $(\widetilde{P}, \mathscr{G})$-Brownian motion. Therefore we can rewrite $(1)$ as follows:

$$
\begin{aligned}
d S_{t}=S_{t-}\left[\left(r+n \lambda I_{t \leq \tau}\right) d t+\sigma_{t} d \widetilde{W}_{t}-\gamma_{t} d D_{t}\right] & \\
& 0 \leq t \leq T,
\end{aligned}
$$

That is, by changing measure, the dynamics (3)-(5) for asset price $S_{t}$ under the physical measure $P$ can be transformed into the following form under the equivalent martingale measure $\widetilde{P}$ :

$$
\begin{aligned}
d S_{t}^{F} & =S_{t}^{F}\left((r+\lambda n) d t+\sigma_{1} d \widetilde{W}_{t}\right), \quad 0 \leq t<\tau, \\
d S_{t}^{d}(\tau) & =S_{t}^{d}(\tau)\left(r d t+\sigma_{2} d \widetilde{W}_{t}\right), \quad \tau<t \leq T, \\
S_{\tau}^{d}(\tau) & =S_{\tau-}^{F}(1-\gamma),
\end{aligned}
$$


where $n=E(\gamma)$. In practice, we may assume that $\gamma$ is a discrete random variable to simplify the computation; for example, we may assume that $\gamma$ takes value $\gamma_{i}$ with probability $p_{i}$ for $i=$ 1,2 , where $0<\gamma_{1} \leq 1$ (loss) and $\gamma_{2}=0$ (no change).

\section{Analytic Formula for Pricing Lookback Options}

In this section, we derive an analytical formula for pricing a floating strike lookback option, whose payoff is the difference between the maximum asset price over the life time and the asset price at expiration.

Using Ito's lemma, the solution of the SDEs (10) can be written as

$$
\begin{aligned}
S_{t}^{F} & =S_{0} e^{\sigma_{1} \widehat{W}_{t}^{F}}, \quad 0 \leq t<\tau, \\
S_{t}^{d}(\tau) & =S_{\tau}^{d}(\tau) e^{\sigma_{2}\left(\widehat{W}_{t}^{d}-\widehat{W}_{\tau}^{d}\right)}, \quad \tau<t \leq T, \\
S_{\tau}^{d}(\tau) & =S_{\tau-}^{F}(1-\gamma),
\end{aligned}
$$

where

$$
\begin{aligned}
\widehat{W}_{t}^{F} & \equiv \alpha_{1} t+\widetilde{W}_{t}, \\
\widehat{W}_{t}^{d} & \equiv \alpha_{2} t+\widetilde{W}_{t}, \\
\alpha_{1} & \equiv \frac{1}{\sigma_{1}}\left(r+\lambda n-\frac{\sigma_{1}^{2}}{2}\right), \\
\alpha_{2} & \equiv \frac{1}{\sigma_{2}}\left(r-\frac{\sigma_{2}^{2}}{2}\right) .
\end{aligned}
$$

Denote the maximum of the asset price up to time $T$ by

$$
Y_{T}=\max _{0 \leq t \leq T} S_{t}
$$

Then at time 0 , the risk-neutral price of the lookback option is given by

$$
V_{0}=E\left[e^{-r T}\left(Y_{T}-S_{T}\right)\right]=E\left[e^{-r T} Y_{T}\right]-E\left[e^{-r T} S_{T}\right] .
$$

Theorem 1. The risk-neutral price of the lookback option at time 0 under models (3)-(5) is given by

$$
\begin{aligned}
V_{0} & =S_{0} e^{(\lambda n-\lambda) T}\left[1+\frac{\sigma_{1}^{2}}{2(r+\lambda n)}\right] \Phi\left(\left(\alpha_{1}+\sigma_{1}\right) \sqrt{T}\right)+S_{0} e^{-(r+\lambda) T}\left[1-\frac{\sigma_{1}^{2}}{2(r+\lambda n)}\right]\left[1-\Phi\left(\alpha_{1} \sqrt{T}\right)\right]+S_{0} e^{-r T} \sum_{i=1}^{2} p_{i} \\
& \cdot \int_{0}^{T} \lambda e^{-\lambda t}\left\{\int_{0}^{\infty} \int_{w}^{\infty}\left[e^{\sigma_{1} m} \Phi\left(\widetilde{d}_{1}^{+}\right)+\left(1-\gamma_{i}\right)\left(1+\frac{\sigma_{2}^{2}}{2 r}\right) e^{\sigma_{1} w+r(T-t)} \Phi\left(\widetilde{d}_{2}\right)-\frac{\sigma_{2}^{2}}{2 r}\left(1-\gamma_{i}\right)^{\left(\sigma_{2}^{2}-2 r\right) / \sigma_{2}^{2}} e^{\sigma_{1} w+\left(2 r \sigma_{1} / \sigma_{2}^{2}\right)(m-w)} \Phi\left(\widetilde{d}_{1}^{-}\right)\right]\right. \\
& \left.\cdot \frac{2(2 m-w)}{t \sqrt{2 \pi t}} e^{\alpha_{1} w-(1 / 2) \alpha_{1}^{2} t-(1 / 2 t)(2 m-w)^{2}} d m d w\right\} d t-S_{0},
\end{aligned}
$$

where $\Phi$ is the distribution function of the standard normal random variable, and

$$
\begin{aligned}
& \beta_{1}=r+\lambda n-\frac{\sigma_{1}^{2}}{2}, \\
& \beta_{2}=r-\frac{\sigma_{2}^{2}}{2}, \\
& \widetilde{d}_{1}^{ \pm}=\frac{ \pm\left[\sigma_{1}(m-w)-\ln \left(1-\gamma_{i}\right)\right]-\beta_{2}(T-t)}{\sigma_{2} \sqrt{T-t}}, \\
& \widetilde{d}_{2}=\frac{-\sigma_{1}(m-w)+\ln \left(1-\gamma_{i}\right)+\left(\beta_{2}+\sigma_{2}^{2}\right)(T-t)}{\sigma_{2} \sqrt{T-t}} .
\end{aligned}
$$

Proof. The risk-neutral price of the lookback option (14) can be rewritten as

$$
\begin{aligned}
V_{0}= & E\left[e^{-r T} Y_{T} \mathbf{1}_{\{\tau \geq T\}}\right]+E\left[e^{-r T} Y_{T} \mathbf{1}_{\{\tau<T\}}\right] \\
& -E\left[e^{-r T} S_{T}\right] .
\end{aligned}
$$

The distribution function of the random variable $S_{T}$ is given by $[10]$

$$
\begin{aligned}
F(S) & =\widetilde{P}\left(S_{T} \leq S\right)=e^{-\lambda T} \Phi\left(\frac{\ln \left(S / S_{0}\right)-a(T)}{b(T)}\right) \\
& +\sum_{i=1}^{2} p_{i} \\
& \cdot \int_{0}^{T} \lambda e^{-\lambda t} \Phi\left(\frac{1}{b(t)} \ln \left(\frac{S}{S_{0}\left(1-\gamma_{i}\right)}\right)-a(t)\right) d t
\end{aligned}
$$

with

$$
\begin{aligned}
& a(t)=\left(r+\lambda n-\frac{\sigma_{1}^{2}}{2}\right) t+\left(r-\frac{1}{2} \sigma_{2}^{2}\right)(T-t), \\
& b(t)=\sqrt{\sigma_{1}^{2} t+\sigma_{2}^{2}(T-t)} .
\end{aligned}
$$

Using the distribution function $F(S)$ and the following identity

$$
\int_{0}^{\infty} S d \Phi\left[\frac{1}{B}\left(\ln \left(\frac{S}{C}\right)-A\right)\right]=C e^{A+B^{2} / 2}
$$


we can compute

$$
\begin{aligned}
& E\left[e^{-r T} S_{T}\right]=S_{0} e^{-r T}\left[e^{(r+\lambda n-\lambda) T}\right. \\
& \left.\quad+\sum_{i=1}^{2} p_{i} \int_{0}^{T} \lambda\left(1-\gamma_{i}\right) e^{(\lambda n-\lambda) t+r T} d t\right]=S_{0} .
\end{aligned}
$$

Next we aim to calculate

$$
E\left[e^{-r T} Y_{T}\right]=E\left[e^{-r T} Y_{T} \mathbf{1}_{\{\tau \geq T\}}\right]+E\left[e^{-r T} Y_{T} \mathbf{1}_{\{\tau<T\}}\right] .
$$

Define

$$
\begin{aligned}
\widehat{M}_{T}^{F} & \equiv \max _{0 \leq t \leq T} \widehat{W}_{t}^{F}, \\
Y_{T}^{F} & \equiv \max _{0 \leq t \leq T} S_{t}^{F} .
\end{aligned}
$$

Then we have

$$
Y_{T}^{F}=\max _{0 \leq t \leq T} S_{0} e^{\sigma_{1} \widehat{W}_{t}^{F}}=S_{0} e^{\sigma_{1} \widehat{M}_{T}^{F}} .
$$

Hence the first term of the right-hand side of (22) can be calculated as

$$
\begin{aligned}
E & {\left[e^{-r T} Y_{T} \mathbf{1}_{\{\tau \geq T\}}\right] } \\
& =e^{-r T} \int_{0}^{\infty} \lambda e^{-\lambda t} E\left[Y_{T} \mathbf{1}_{\{\tau \geq T\}} \mid \tau=t\right] d t \\
& =e^{-r T} \int_{T}^{\infty} \lambda e^{-\lambda t} E\left[Y_{T}^{F} \mid \tau=t\right] d t \\
& =e^{-(r+\lambda) T} E\left[Y_{T}^{F}\right] .
\end{aligned}
$$

According to [12], the density under $\widetilde{P}$ for the random variable $\widehat{M}_{T}^{F}$ is given by

$$
\begin{aligned}
\widetilde{f}_{\widehat{M}_{T}^{F}}(m)= & \frac{2}{\sqrt{2 \pi T}} e^{-(1 / 2 T)\left(m-\alpha_{1} T\right)^{2}} \\
& -2 \alpha_{1} e^{2 \alpha_{1} m} \Phi\left(\frac{-m-\alpha_{1} T}{\sqrt{T}}\right), \quad m \geq 0 .
\end{aligned}
$$

So we have

$$
\begin{aligned}
& E\left[Y_{T}^{F}\right]=\int_{0}^{\infty} S_{0} e^{\sigma_{1} m}\left[\frac{2}{\sqrt{2 \pi T}} e^{-(1 / 2 T)\left(m-\alpha_{1} T\right)^{2}}\right. \\
& \left.-2 \alpha_{1} e^{2 \alpha_{1} m} \Phi\left(\frac{-m-\alpha_{1} T}{\sqrt{T}}\right)\right] d m \\
& =2 S_{0} e^{(r+\lambda n) T} \int_{0}^{\infty} \frac{1}{\sqrt{2 \pi T}} e^{-\left[m-\left(\alpha_{1}+\sigma_{1}\right) T\right]^{2} / 2 T} d m \\
& -S_{0}\left[1-\frac{\sigma_{1}^{2}}{2(r+\lambda n)}\right] \\
& \cdot \int_{0}^{\infty} \Phi\left(\frac{-m-\alpha_{1} T}{\sqrt{T}}\right) d\left(e^{\left(\sigma_{1}+2 \alpha_{1}\right) m}\right) \\
& =2 S_{0} e^{(r+\lambda n) T} \Phi\left(\left(\alpha_{1}+\sigma_{1}\right) \sqrt{T}\right)-S_{0}[1
\end{aligned}
$$

$$
\begin{aligned}
& \left.-\frac{\sigma_{1}^{2}}{2(r+\lambda n)}\right]\left[-\Phi\left(-\alpha_{1} \sqrt{T}\right)\right. \\
& \left.+e^{(r+\lambda n) T} \Phi\left(\left(\alpha_{1}+\sigma_{1}\right) \sqrt{T}\right)\right]=S_{0} e^{(r+\lambda n) T}[1 \\
& \left.+\frac{\sigma_{1}^{2}}{2(r+\lambda n)}\right] \Phi\left(\left(\alpha_{1}+\sigma_{1}\right) \sqrt{T}\right)+S_{0}[1 \\
& \left.-\frac{\sigma_{1}^{2}}{2(r+\lambda n)}\right]\left[1-\Phi\left(\alpha_{1} \sqrt{T}\right)\right] .
\end{aligned}
$$

Hence the value of the expectation in (25) can be calculated as

$$
\begin{aligned}
& E\left[e^{-r T} Y_{T} \mathbf{1}_{\{\tau \geq T\}}\right] \\
& =S_{0} e^{(\lambda n-\lambda) T}\left[1+\frac{\sigma_{1}^{2}}{2(r+\lambda n)}\right] \Phi\left(\left(\alpha_{1}+\sigma_{1}\right) \sqrt{T}\right) \\
& \quad+S_{0} e^{-(r+\lambda) T}\left[1-\frac{\sigma_{1}^{2}}{2(r+\lambda n)}\right]\left[1-\Phi\left(\alpha_{1} \sqrt{T}\right)\right] .
\end{aligned}
$$

Denote

$$
\begin{aligned}
\widehat{M}_{T-t}^{d} & \equiv \max _{0 \leq t \leq T-t} \widehat{W}_{t}^{d}, \\
Y_{t}^{d} & \equiv \max _{t \leq s \leq T} S_{s}^{d}(t) .
\end{aligned}
$$

Then

$$
\begin{aligned}
Y_{t}^{d} & =\max _{t \leq s \leq T} S_{t}^{d}(t) e^{\sigma_{2}\left(\widehat{W}_{s}^{d}-\widehat{W}_{t}^{d}\right)}, \\
\frac{Y_{t}^{d}}{S_{t}^{d}(t)} & =e^{\max _{t \leq s \leq T} \sigma_{2}\left(\widehat{W}_{s}^{d}-\widehat{W}_{t}^{d}\right)},
\end{aligned}
$$

which is independent of $\mathscr{F}_{t}$. Then we calculate the second term of the right-hand side of (22) as

$$
\begin{gathered}
E\left[e^{-r T} Y_{T} \mathbf{1}_{\{\tau<T\}}\right]=e^{-r T} E\left[\max \left(Y_{\tau}^{F}, Y_{\tau}^{d}\right) \mathbf{1}_{\{\tau<T\}}\right] \\
=e^{-r T} \int_{0}^{T} \lambda e^{-\lambda t} E\left[\max \left(Y_{\tau}^{F}, Y_{\tau}^{d}\right) \mid \tau=t\right] d t \\
=e^{-r T} \int_{0}^{T} \lambda e^{-\lambda t} E\left[\sum_{i=1}^{2} p_{i} S_{t}^{d}(t)\right. \\
\left.\cdot E\left[\max \left(\frac{Y_{t}^{F}}{S_{t}^{d}(t)}, \frac{Y_{t}^{d}}{S_{t}^{d}(t)}\right) \mid \mathscr{F}_{t}, \gamma\right]\right] d t .
\end{gathered}
$$

We shall use the independence lemma (see [12]) to calculate the above expectations. To this end, we define

$$
\begin{aligned}
G(t, x) & =E\left[\max \left(x, \frac{Y_{t}^{d}}{S_{t}^{d}(t)}\right) \mid \gamma=\gamma_{i}\right], \\
R(T-t, m) & =\widetilde{P}\left\{\widehat{M}_{T-t}^{d} \leq m\right\} .
\end{aligned}
$$


According to [12], we have

$$
\begin{aligned}
\widetilde{P}\left\{\widehat{M}_{T-t}^{d} \leq m\right\}= & \Phi\left[\frac{m-\alpha_{2}(T-t)}{\sqrt{T-t}}\right] \\
& -e^{2 \alpha_{2} m} \Phi\left[\frac{-m-\alpha_{2}(T-t)}{\sqrt{T-t}}\right],
\end{aligned}
$$

$m \geq 0$.

Then

$$
\begin{aligned}
G( & , x)=E\left[\max \left(x, \frac{Y_{t}^{d}}{S_{t}^{d}(t)}\right) \mid \gamma=\gamma_{i}\right] \\
& =E\left[\exp \left\{\max \left(\ln x, \max _{t \leq s \leq T} \sigma_{2}\left(\widehat{W}_{s}^{d}-\widehat{W}_{t}^{d}\right)\right)\right\} \mid \gamma\right. \\
& \left.=\gamma_{i}\right]=E\left[\exp \left\{\max \left(\ln x, \sigma_{2} \max _{0 \leq s \leq T-t} \widehat{W}_{s}^{d}\right)\right\} \mid \gamma\right. \\
& \left.=\gamma_{i}\right]=E\left[\exp \left\{\max \left(\ln x, \sigma_{2} \widehat{M}_{T-t}^{d}\right)\right\} \mid \gamma=\gamma_{i}\right] \\
& =E\left[x \mathbf{1}_{\left\{\ln x>\sigma_{2} \widehat{M}_{T-t}^{d}\right\}} \mid \gamma=\gamma_{i}\right] \\
& +E\left[e^{\sigma_{2} \widehat{M}_{T-t}^{d}} \mathbf{1}_{\left\{\ln x \leq \sigma_{2} \widehat{M}_{T-t}^{d}\right\}} \mid \gamma=\gamma_{i}\right]=x R(T \\
& \left.-t, \frac{1}{\sigma_{2}} \ln x\right)+\int_{(\ln x) / \sigma_{2}}^{\infty} e^{\sigma_{2} m} R_{m}(T-t, m) d m \\
& =x\left[\Phi\left(d_{1}^{+}\right)-x^{2 \beta_{2} / \sigma_{2}^{2}} \Phi\left(d_{1}^{-}\right)\right]+\left(1+\frac{\sigma_{2}^{2}}{2 r}\right) \\
& \left.+\frac{\sigma_{2}^{2}}{2 r}\right) e^{r(T-t)} \Phi\left(d_{2}\right)-\frac{\sigma_{2}^{2}}{2 r} x^{2 r / \sigma_{2}^{2}} \Phi\left(d_{1}^{-}\right), \\
& e^{r(T-t)} \Phi\left(d_{2}\right)+\frac{\beta_{2}}{r} x^{2 r / \sigma_{2}^{2}} \Phi\left(d_{1}^{-}\right)=x \Phi\left(d_{1}^{+}\right)+(1
\end{aligned}
$$

where

$$
\begin{aligned}
& \beta_{2} \equiv r-\frac{\sigma_{2}^{2}}{2}, \\
& d_{1}^{ \pm} \equiv \frac{ \pm \ln (x)-\beta_{2}(T-t)}{\sigma_{2} \sqrt{T-t}}, \\
& d_{2} \equiv \frac{-\ln (x)+\left(\beta_{2}+\sigma_{2}^{2}\right)(T-t)}{\sigma_{2} \sqrt{T-t}} .
\end{aligned}
$$

By the independence lemma (see [12]), we have

$$
E\left[\max \left(\frac{Y_{t}^{F}}{S_{t}^{d}(t)}, \frac{Y_{t}^{d}}{S_{t}^{d}(t)}\right) \mid \mathscr{F}_{t}, \gamma\right]=G\left(t, \frac{Y_{t}^{F}}{S_{t}^{d}(t)}\right) .
$$

According to [12], the joint density function under $\widetilde{P}$ of $\left(\widehat{W}_{t}^{F}\right.$, $\widehat{M}_{t}^{F}$ ) involved in $Y_{t}^{F}$ and $S_{t}^{d}(t)$ (see (10)) is

$$
\widetilde{f}_{\widehat{W}_{t}^{F}, \widehat{M}_{t}^{F}}(m, w)=\frac{2(2 m-w)}{t \sqrt{2 \pi t}} e^{\alpha_{1} w-(1 / 2) \alpha_{1}^{2} t-(1 / 2 t)(2 m-w)^{2}},
$$

Substituting (38) and (36) into (32) and using (39), we derive that

$$
\begin{aligned}
E[ & \left.e^{-r T} Y_{T} \mathbf{1}_{\{\tau<T\}}\right]=e^{-r T} \int_{0}^{T} \lambda e^{-\lambda t} \\
& . \sum_{i=1}^{2} p_{i} E\left[Y_{t}^{F} \Phi\left(d_{1}^{+}\right)+S_{t}^{d}(t)\left(1+\frac{\sigma_{2}^{2}}{2 r}\right) e^{r(T-t)} \Phi\left(d_{2}\right)\right. \\
& \left.-S_{t}^{d}(t) \frac{\sigma_{2}^{2}}{2 r}\left(\frac{Y_{t}^{F}}{S_{t}^{d}(t)}\right)^{2 r / \sigma_{2}^{2}} \Phi\left(d_{1}^{-}\right)\right] d t \\
& =S_{0} e^{-r T} \sum_{i=1}^{2} p_{i} \int_{0}^{T} \lambda e^{-\lambda t}\left\{\int _ { 0 } ^ { \infty } \int _ { w } ^ { \infty } \left[e^{\sigma_{1} m} \Phi\left(\widetilde{d}_{1}^{+}\right)\right.\right. \\
& +\left(1-\gamma_{i}\right)\left(1+\frac{\sigma_{2}^{2}}{2 r}\right) e^{\sigma_{1} w+r(T-t)} \Phi\left(\widetilde{d}_{2}\right) \\
& \left.-\frac{\sigma_{2}^{2}}{2 r}\left(1-\gamma_{i}\right)^{\left(\sigma_{2}^{2}-2 r\right) / \sigma_{2}^{2}} e^{\sigma_{1} w+\left(2 r \sigma_{1} / \sigma_{2}^{2}\right)(m-w)} \Phi\left(\widetilde{d}_{1}^{-}\right)\right] \\
& \left.+\frac{2(2 m-w)}{t \sqrt{2 \pi t}} e^{\alpha_{1} w-(1 / 2) \alpha_{1}^{2} t-(1 / 2 t)(2 m-w)^{2}} d m d w\right\} d t
\end{aligned}
$$

where $\widetilde{d}_{1}^{ \pm}$and $\widetilde{d}_{2}$ are defined in Theorem 1 . Combining (28), (40), and (22) gives the value of $E\left[e^{-r T} Y_{T}\right]$, and then using (21) completes the proof of this theorem.

\section{Analytic Formula for Pricing Barrier Options}

In this section we derive an analytic formula for pricing barrier options under the counterparty risk models (10). The barrier options include up-and-out, up-and-in, down-andout, and down-and-in puts and calls. Since the approaches for deriving the formulas for pricing these kinds of barrier options are similar, we only study the up-and-out barrier call in this section.

Consider an up-and-out barrier call option with expiry date $T$, strike price $K$, and barrier level $B$ and we assume that $K<B$. The solution to the stochastic differential equation for the asset price is given by (11), and the maximum of the asset price $Y_{T}$ is defined by (13). The option knocks out (i.e., pays off 0 ) if and only if $Y_{T}>B$. If $Y_{T} \leq B$, then the option pays off $\left(S_{T}-K\right)^{+}$. In order words, the payoff of the option is

$$
V_{T}^{u o}=\left(S_{T}-K\right)^{+} \mathbf{1}_{\left\{Y_{T} \leq B\right\}}=\left(S_{T}-K\right) \mathbf{1}_{\left\{S_{T} \geq K, Y_{T} \leq B\right\}} \cdot
$$

At time 0 , the risk-neutral price of the up-and-out barrier call option is

$$
V_{0}^{u o}=E\left[e^{-r T}\left(S_{T}-K\right) \mathbf{1}_{\left\{S_{T} \geq K, Y_{T} \leq B\right\}}\right] .
$$


Theorem 2. The risk-neutral price of an up-and-out barrier call option at time 0 under models (3)-(5) is given by

$$
\begin{aligned}
V_{0}^{u o} & =e^{-\lambda T} \widetilde{V}_{0}^{u o}+\sum_{i=1}^{2} p_{i} \\
\cdot & \int_{0}^{T} \lambda e^{-(\lambda+r) t} \int_{0}^{\left(1 / \sigma_{1}\right) \ln \left(B / S_{0}\right)} \int_{w}^{\left(1 / \sigma_{1}\right) \ln \left(B / S_{0}\right)}\left\{\varphi\left(\gamma_{i}, w\right)\right. \\
\cdot & {\left[\Phi\left(\widehat{d}_{2}^{+}\left(\frac{\varphi\left(\gamma_{i}, w\right)}{K}\right)\right)-\Phi\left(\hat{d}_{2}^{+}\left(\frac{\varphi\left(\gamma_{i}, w\right)}{B}\right)\right)\right] } \\
& -e^{-r(T-t)} K\left[\Phi\left(\hat{d}_{2}^{-}\left(\frac{\varphi\left(\gamma_{i}, w\right)}{K}\right)\right)-\Phi\left(\hat{d}_{2}^{-}\left(\frac{\varphi\left(\gamma_{i}, w\right)}{B}\right)\right)\right] \\
& -B\left[\frac{\varphi\left(\gamma_{i}, w\right)}{B}\right]^{-2 r / \sigma_{2}^{2}}\left[\Phi\left(\hat{d}_{2}^{+}\left(\frac{B^{2}}{K \varphi\left(\gamma_{i}, w\right)}\right)\right)-\Phi\left(\hat{d}_{2}^{+}\left(\frac{B}{\varphi\left(\gamma_{i}, w\right)}\right)\right)\right] \\
+ & e^{-r(T-t)} K\left[\frac{\varphi\left(\gamma_{i}, w\right)}{B}\right]^{1-2 r / \sigma_{2}^{2}} \\
\cdot & {\left.\left[\Phi\left(\hat{d}_{2}^{-}\left(\frac{\varphi\left(\gamma_{i}, w\right)}{K}\right)\right)-\Phi\left(\hat{d}_{2}^{-}\left(\frac{\varphi\left(\gamma_{i}, w\right)}{B}\right)\right)\right]\right\} } \\
& \cdot \frac{2(2 m-w)}{t \sqrt{2 \pi t}} e^{\alpha_{1} w-(1 / 2) \alpha_{1}^{2} t-(1 / 2 t)(2 m-w)^{2}} d m d w d t,
\end{aligned}
$$

where

$$
\begin{aligned}
\widetilde{V}_{0}^{u o}= & S_{0} e^{\lambda n T}\left[\Phi\left(\hat{d}_{1}^{+}\left(\frac{S_{0}}{K}\right)\right)-\Phi\left(\hat{d}_{1}^{+}\left(\frac{S_{0}}{B}\right)\right)\right] \\
& -e^{-r T} K\left[\Phi\left(\hat{d}_{1}^{-}\left(\frac{S_{0}}{K}\right)\right)-\Phi\left(\hat{d}_{1}^{-}\left(\frac{S_{0}}{B}\right)\right)\right] \\
& -B\left(\frac{S_{0}}{B}\right)^{-2(r+\lambda n) / \sigma_{1}^{2}} \\
& \cdot\left[\Phi\left(\hat{d}_{1}^{+}\left(\frac{B^{2}}{K S_{0}}\right)\right)-\Phi\left(\hat{d}_{1}^{+}\left(\frac{B}{S_{0}}\right)\right)\right] \\
+ & e^{-r T} K\left(\frac{S_{0}}{B}\right)^{-\left(2(r+\lambda n) / \sigma_{1}^{2}\right)+1} \\
& \cdot\left[\Phi\left(\hat{d}_{1}^{-}\left(\frac{S_{0}}{K}\right)\right)-\Phi\left(\hat{d}_{1}^{-}\left(\frac{S_{0}}{B}\right)\right)\right],
\end{aligned}
$$

with

$$
\begin{aligned}
\widehat{d}_{1}^{ \pm}(s) & =\frac{1}{\sigma_{1} \sqrt{T}}\left[\ln (s)+\left(r+\lambda n \pm \frac{1}{2} \sigma_{1}^{2}\right) T\right], \\
\widehat{d}_{2}^{ \pm}(s) & =\frac{1}{\sigma_{2} \sqrt{T-t}}\left[\ln (s)+\left(r \pm \frac{1}{2} \sigma_{2}^{2}\right)(T-t)\right], \\
\varphi\left(\gamma_{i}, w\right) & =S_{0}\left(1-\gamma_{i}\right) e^{\sigma_{1} w} .
\end{aligned}
$$

Proof. We rewrite (42) as follows:

$$
\begin{aligned}
V_{0}^{u o}= & E\left[e^{-r T}\left(S_{T}-K\right) \mathbf{1}_{\left\{\left\{S_{T} \geq K, Y_{T} \leq B\right\}\right\}} \mathbf{1}_{\{\tau>T\}}\right] \\
& +E\left[e^{-r T}\left(S_{T}-K\right) \mathbf{1}_{\left\{\left\{S_{T} \geq K, Y_{T} \leq B\right\}\right\}} \mathbf{1}_{\{\tau \leq T\}}\right] .
\end{aligned}
$$

The first term on the right-hand side of (46) is calculated as

$$
\begin{aligned}
E & {\left[e^{-r T}\left(S_{T}-K\right) \mathbf{1}_{\left\{\left\{S_{T} \geq K, Y_{T} \leq B\right\}\right\}} \mathbf{1}_{\{\tau>T\}}\right] } \\
& =e^{-\lambda T} E\left[e^{-r T}\left(S_{T}^{F}-K\right) \mathbf{1}_{\left\{\left\{S_{T}^{F} \geq K, Y_{T}^{F} \leq B\right\}\right\}}\right]=: e^{-\lambda T} \widetilde{V}_{0}^{u o} .
\end{aligned}
$$

Notice that $\widetilde{V}_{0}^{u o}$ corresponds to the case when there is no default of the counterparty. Then we can calculate it using a similar technique as in [12] and obtain that

$$
\begin{aligned}
\widetilde{V}_{0}^{u o}= & S_{0} e^{\lambda n T}\left[\Phi\left(\hat{d}_{1}^{+}\left(\frac{S_{0}}{K}\right)\right)-\Phi\left(\hat{d}_{1}^{+}\left(\frac{S_{0}}{B}\right)\right)\right] \\
- & e^{-r T} K\left[\Phi\left(\hat{d}_{1}^{-}\left(\frac{S_{0}}{K}\right)\right)-\Phi\left(\hat{d}_{1}^{-}\left(\frac{S_{0}}{B}\right)\right)\right] \\
- & B\left(\frac{S_{0}}{B}\right)^{-2(r+\lambda n) / \sigma_{1}^{2}} \\
& \cdot\left[\Phi\left(\widehat{d}_{1}^{+}\left(\frac{B^{2}}{K S_{0}}\right)\right)-\Phi\left(\hat{d}_{1}^{+}\left(\frac{B}{S_{0}}\right)\right)\right] \\
+ & e^{-r T} K\left(\frac{S_{0}}{B}\right)^{-\left(2(r+\lambda n) / \sigma_{1}^{2}\right)+1} \\
& \cdot\left[\Phi\left(\hat{d}_{1}^{-}\left(\frac{S_{0}}{K}\right)\right)-\Phi\left(\hat{d}_{1}^{-}\left(\frac{S_{0}}{B}\right)\right)\right],
\end{aligned}
$$

where $\widehat{d}_{1}^{ \pm}(s)$ are defined in Theorem 2 .

Then we compute the second term of (46):

$$
\begin{aligned}
E & {\left[e^{-r T}\left(S_{T}-K\right) \mathbf{1}_{\left\{\left\{S_{T} \geq K, Y_{T} \leq B\right\}\right\}} \mathbf{1}_{\{\tau \leq T\}}\right] } \\
& =\int_{0}^{T} \lambda e^{-\lambda t} E\left[e^{-r T}\left(S_{T}-K\right) \mathbf{1}_{\left\{S_{T} \geq K, Y_{\tau}^{F} \leq B, Y_{\tau}^{d} \leq B\right\}} \mid \tau\right. \\
& =t] d t \\
& =\int_{0}^{T} \lambda e^{-\lambda t} E\left[\mathbf { 1 } _ { \{ Y _ { t } ^ { F } \leq B \} } E \left[e^{-r T}\left(S_{T}^{d}(t)-K\right)\right.\right. \\
& \left.\left.\cdot \mathbf{1}_{\left\{S_{T}^{d}(t) \geq K, Y_{t}^{d} \leq B\right\}} \mid \mathscr{F}_{t}\right]\right] d t .
\end{aligned}
$$

Using the definitions of (11) and (31), we have

$$
\begin{aligned}
S_{t}^{d}(t) & =S_{0}(1-\gamma) e^{\sigma_{1} \widehat{W}_{t}^{F}}, \\
\frac{S_{T}^{d}(t)}{S_{t}^{d}(t)} & =e^{\sigma_{2}\left(\widehat{W}_{T}^{d}-\widehat{W}_{t}^{d}\right)}, \\
\frac{Y_{t}^{d}}{S_{t}^{d}(t)} & =e^{\sigma_{2} \max _{t \leq s \leq T}\left(\widehat{W}_{s}^{d}-\widehat{W}_{t}^{d}\right)} .
\end{aligned}
$$

It is obvious that $S_{T}^{d}(t) / S_{t}^{d}(t)$ and $Y_{t}^{d} / S_{t}^{d}(t)$ are independent on $\mathscr{F}_{t}$. Letting $x=S_{t}^{d}(t)$, we calculate

$$
\begin{aligned}
& E\left[e^{-r T}\left(S_{T}^{d}(t)-K\right) \mathbf{1}_{\left\{S_{T}^{d}(t) \geq K, Y_{t}^{d} \leq B\right\}} \mid \mathscr{F}_{t}\right] \\
& =\sum_{i=1}^{2} p_{i} E\left[e^{-r T}\left(x e^{\sigma_{2}\left(\widehat{W}_{T}^{d}-\widehat{W}_{t}^{d}\right)}-K\right)\right. \\
& \left.\cdot \mathbf{1}_{\left\{x e^{\sigma_{2}\left(\widehat{W}_{T}^{d}-\widehat{W}_{t}^{d}\right)} \geq K, x e^{\left.\sigma_{2} \max _{t \leq s \leq T}\left(\widehat{W}_{s}^{d}-\widehat{W}_{t}^{d}\right) \leq B\right\}}\right.} \mid \gamma=\gamma_{i}\right] \\
& =\sum_{i=1}^{2} p_{i} E\left[e^{-r T}\left(x e^{\sigma_{2} \widehat{W}_{T-t}^{d}}-K\right)\right.
\end{aligned}
$$




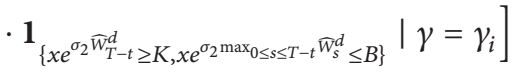

$$
\begin{aligned}
& =\sum_{i=1}^{2} p_{i} e^{-r t} E\left[e^{-r(T-t)}\left(x e^{\sigma_{2} \widehat{W}_{T-t}^{d}}-K\right)\right. \\
& \left.\cdot \mathbf{1}_{\left\{\widehat{W}_{T-t}^{d} \geq k, \widehat{M}_{T-t}^{d} \leq b\right\}} \mid \gamma=\gamma_{i}\right],
\end{aligned}
$$

where

$$
\begin{aligned}
& k=\frac{1}{\sigma_{2}} \ln \frac{K}{x}, \\
& b=\frac{1}{\sigma_{2}} \ln \frac{B}{x} .
\end{aligned}
$$

Notice that the expectation

$$
E\left[e^{-r(T-t)}\left(x e^{\sigma_{2} \widehat{W}_{T-t}^{d}}-K\right) \mathbf{1}_{\left\{\widehat{W}_{T-t}^{d} \geq k, \widehat{M}_{T-t}^{d} \leq b\right\}} \mid \gamma=\gamma_{i}\right]
$$

corresponds to the case when there is no default of the counterparty. Therefore using the techniques in [12], we have

$$
\begin{aligned}
E & {\left[e^{-r(T-t)}\left(x e^{\sigma_{2} \widehat{W}_{T-t}^{d}}-K\right) \mathbf{1}_{\left\{\widehat{W}_{T-t}^{d} \geq k, \widehat{M}_{T-t}^{d} \leq b\right\}} \mid \gamma=\gamma_{i}\right] } \\
& =x\left[\Phi\left(\widehat{d}_{2}^{+}\left(\frac{x}{K}\right)\right)-\Phi\left(\widehat{d}_{2}^{+}\left(\frac{x}{B}\right)\right)\right] \\
& -e^{-r(T-t)} K\left[\Phi\left(\widehat{d}_{2}^{-}\left(\frac{x}{K}\right)\right)-\Phi\left(\hat{d}_{2}^{-}\left(\frac{x}{B}\right)\right)\right] \\
& -B\left(\frac{x}{B}\right)^{-2 r / \sigma_{2}^{2}}\left[\Phi\left(\widehat{d}_{2}^{+}\left(\frac{B^{2}}{K x}\right)\right)-\Phi\left(\widehat{d}_{2}^{+}\left(\frac{B}{x}\right)\right)\right] \\
& +e^{-r(T-t)} K\left(\frac{x}{B}\right)^{-\left(2 r / \sigma_{2}^{2}\right)+1} \\
& \cdot\left[\Phi\left(\hat{d}_{2}^{-}\left(\frac{x}{K}\right)\right)-\Phi\left(\hat{d}_{2}^{-}\left(\frac{x}{B}\right)\right)\right]
\end{aligned}
$$

where $\widehat{d}_{2}^{ \pm}(s)$ are defined in Theorem 2 .

Substituting (54) and $x=S_{t}^{d}(t)$ into (51) and using (39), we can continue to calculate (49) as

$$
\begin{aligned}
E & {\left[e^{-r T}\left(S_{T}-K\right) \mathbf{1}_{\left\{S_{T} \geq K, Y_{T} \leq B\right\}} \mathbf{1}_{\{\tau \leq T\}}\right] } \\
& =\sum_{i=1}^{2} p_{i} \int_{0}^{T} \lambda e^{-(\lambda+r) t} \int_{0}^{\left(1 / \sigma_{1}\right) \ln \left(B / S_{0}\right)} \int_{w}^{\left(1 / \sigma_{1}\right) \ln \left(B / S_{0}\right)}\left\{\varphi\left(\gamma_{i}, w\right)\right. \\
& \cdot\left[\Phi\left(\hat{d}_{2}^{+}\left(\frac{\varphi\left(\gamma_{i}, w\right)}{K}\right)\right)-\Phi\left(\hat{d}_{2}^{+}\left(\frac{\varphi\left(\gamma_{i}, w\right)}{B}\right)\right)\right] \\
& -e^{-r(T-t)} K\left[\Phi\left(\hat{d}_{2}^{-}\left(\frac{\varphi\left(\gamma_{i}, w\right)}{K}\right)\right)-\Phi\left(\hat{d}_{2}^{-}\left(\frac{\varphi\left(\gamma_{i}, w\right)}{B}\right)\right)\right] \\
& -B\left[\frac{\varphi\left(\gamma_{i}, w\right)}{B}\right]^{-2 r / \sigma_{2}^{2}}\left[\Phi\left(\hat{d}_{2}^{+}\left(\frac{B^{2}}{K \varphi\left(\gamma_{i}, w\right)}\right)\right)-\Phi\left(\hat{d}_{2}^{+}\left(\frac{B}{\varphi\left(\gamma_{i}, w\right)}\right)\right)\right] \\
& +e^{-r(T-t)} K\left[\frac{\varphi\left(\gamma_{i}, w\right)}{B}\right]^{1-2 r / \sigma_{2}^{2}} \\
& \left.\cdot\left[\Phi\left(\hat{d}_{2}^{-}\left(\frac{\varphi\left(\gamma_{i}, w\right)}{K}\right)\right)-\Phi\left(\hat{d}_{2}^{-}\left(\frac{\varphi\left(\gamma_{i}, w\right)}{B}\right)\right)\right]\right\} \\
& \cdot \frac{2(2 m-w)}{t \sqrt{2 \pi t}} e^{\alpha_{1} w-(1 / 2) \alpha_{1}^{2} t-(1 / 2 t)(2 m-w)^{2}} d m d w d t
\end{aligned}
$$

where $\varphi\left(\gamma_{i}, w\right)$ is defined in Theorem 2. Combining (47) and (55), we obtain the formula for $V_{0}^{u o}$.

\section{Conclusions}

This paper derives analytic formulas for lookback and barrier options when the underlying asset is subject to counterparty risk. The counterparty default risk induces a drop in the price of the risky asset (stock), and the stock can still be traded after this default time. This kind of counterparty risk causes difficulty in deriving the density of the first passage time for the maximum asset price. The conditional density approach, which is developed by Jiao and Pham [9] for optimal investment, is utilized to overcome the difficulty and derive the formulas for the lookback and barrier options. Future research lies in deriving analytic formulas for the exotic options with two underlying assets exposed to bilateral counterparty risk.

\section{Conflicts of Interest}

The author declares that there are no conflicts of interest regarding the publication of this paper.

\section{Acknowledgments}

The work was supported by Science and Technology Research Projects of Chongqing Education Committee (Grant no. KJ15012004). The author thanks Mr. Xiaosi Shen for making partial contributions to the paper.

\section{References}

[1] F. A. Longstaff, "How much can marketability affect security values?" The Journal of Finance, vol. 50, no. 5, pp. 1767-1774, 1995.

[2] R. C. Merton, "On the pricing of corporate debt: the risk structure of interest rates," The Journal of Finance, vol. 29, no. 2, pp. 449-469, 1974.

[3] F. Black and J. C. Cox, "Valuing corporate securities: some effects of bond indenture provisions," The Journal of Finance, vol. 31, no. 2, pp. 351-367, 1976.

[4] H. E. Leland and K. B. Toft, "Optimal capital structure, endogenous bankruptcy, and the term structure of credit spreads," Journal of Finance, vol. 51, no. 3, pp. 987-1019, 1996.

[5] N. Chen and S. G. Kou, "Credit spreads, optimal capital structure, and implied volatility with endogenous default and jump risk," Mathematical Finance, vol. 19, no. 3, pp. 343-378, 2009.

[6] S. G. Kou, "Discrete barrier and lookback options," in Handbooks in OR and MS, J. Birge and V. Linetsky, Eds., vol. 15, chapter 8, Elsevier, 2008.

[7] R. A. Jarrow and F. Yu, "Counterparty risk and the pricing of defaultable securities," Journal of Finance, vol. 56, no. 5, pp. 1765-1799, 2001.

[8] N. El Karoui, M. Jeanblanc, and Y. Jiao, "What happens after a default: the conditional density approach," Stochastic Processes and Their Applications, vol. 120, no. 7, pp. 1011-1032, 2010.

[9] Y. Jiao and H. Pham, "Optimal investment with counterparty risk: a default-density model approach," Finance and Stochastics, vol. 15, no. 4, pp. 725-753, 2011. 
[10] J. Ma, D. Deng, and H. Zheng, "Convergence analysis and optimal strike choice for static hedges of general path-independent pay-offs," Quantitative Finance, vol. 16, no. 4, pp. 593-603, 2016.

[11] R. Mansuy and M. Yor, Random Times and Enlargements of Filtrations in a Brownian Setting, Springer, 2005.

[12] S. E. Shreve, Stochastic Calculus for Finance II: Continuous-Time Models, Springer, 2004. 


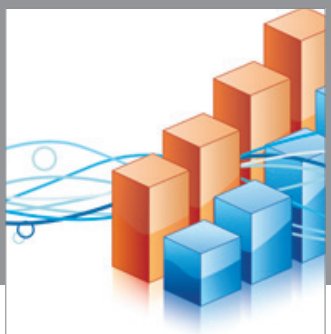

Advances in

Operations Research

vatem alat4

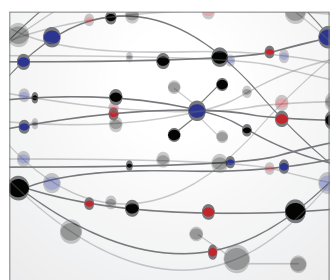

\section{The Scientific} World Journal
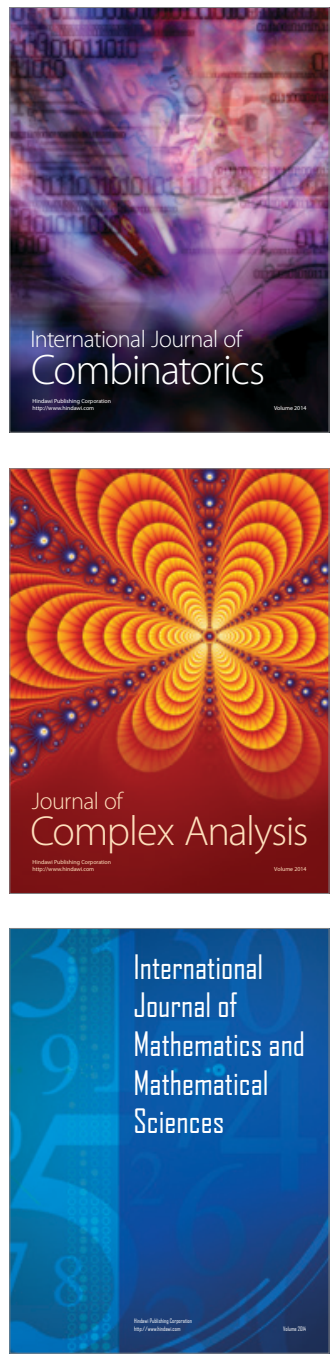
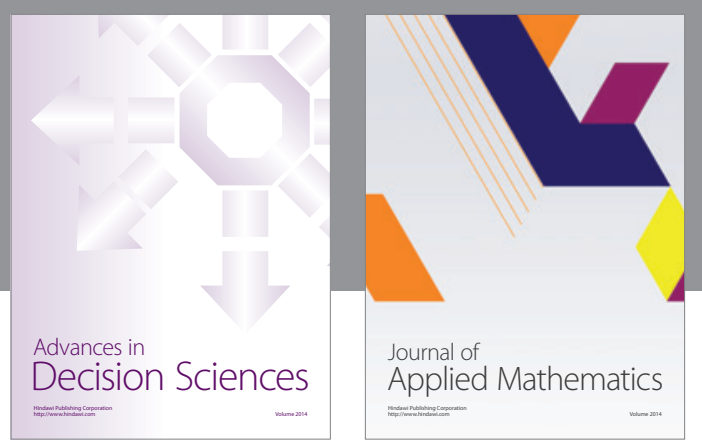

Algebra

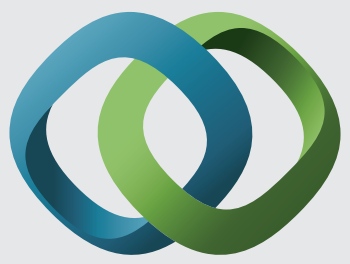

\section{Hindawi}

Submit your manuscripts at

https://www.hindawi.com
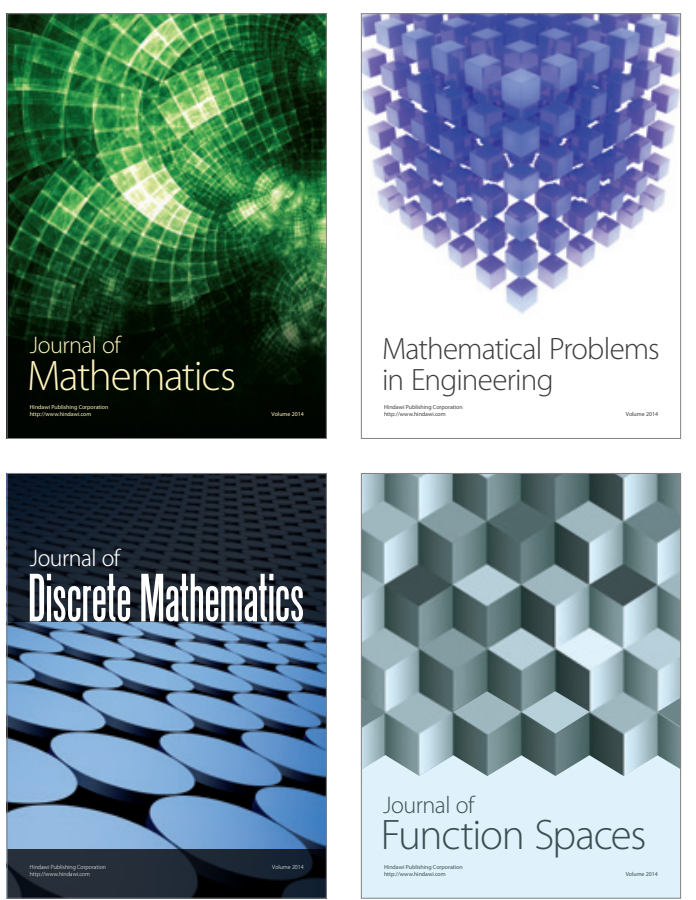

Mathematical Problems in Engineering
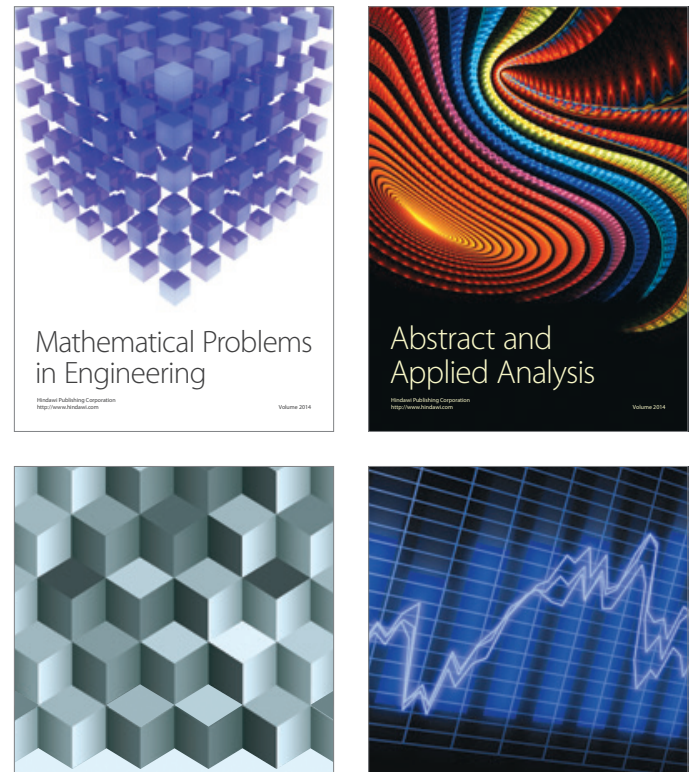

Journal of

Function Spaces

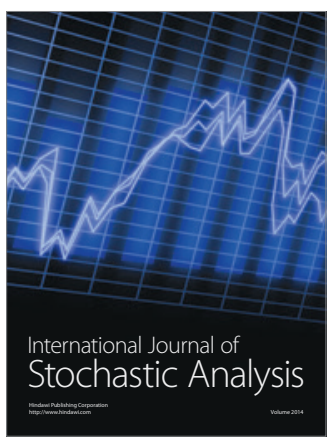

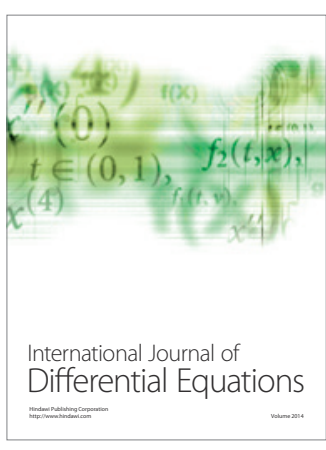
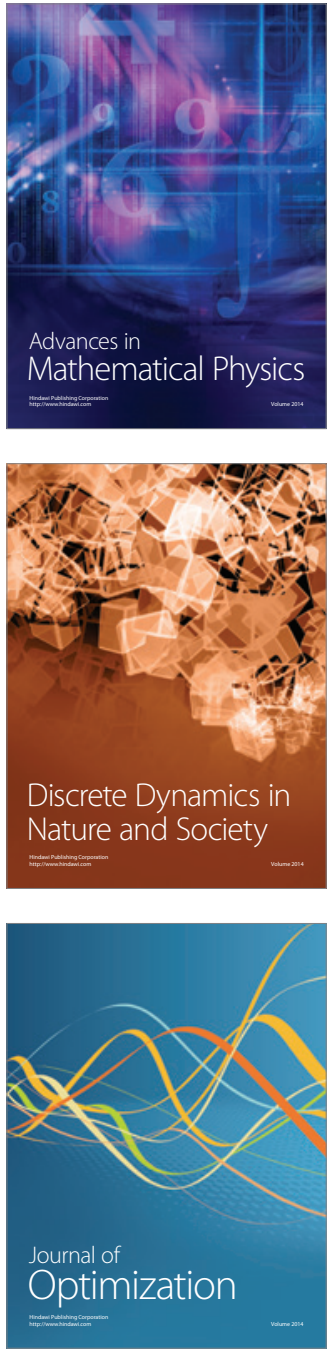